\title{
SPECTRAL CLASSIFICATION OF CARBON-PECULIAR G STARS
}

\author{
TUBA KOKTAY \\ Astronomy and Space Science Department \\ Istanbul University \\ Istanbul, Türkiye \\ AND \\ R. F. GARRISON \\ David Dunlap Observatory \\ University of Toronto \\ Toronto, Canada
}

\begin{abstract}
Spectroscopic observations at classification dispersion have been obtained for a set of stars having abnormal photometric indices on the Strömgren uvby system. Five of the stars observed have extreme carbon anomalies; two of these spectra are illustrated here.
\end{abstract}

\section{Introduction}

During a uvby photometric survey of ten thousand solar-type stars, Olsen $(1993,1994)$ isolated approximately 200 stars with uniquely peculiar photometric indices. We have observed $\sim 120$ of these stars spectroscopically at MK resolution (1-3 $\AA$ ) in order to look for spectral anomalies which might account for their abnormal colors.

\section{Spectroscopic Observations}

The stars were observed with a CCD spectrograph on the 60-cm Helen Sawyer Hogg Telescope of the University of Toronto Southern Observatory on Cerro Las Campanas in Chile. The detector is a PM512 CCD with 20 micron pixels coated with MetaChrome II for improved blue response. The resolution can be either $1.7 \AA(0.85 \AA / \mathrm{pix})$ or $3.4 \AA(1.7 \AA / \mathrm{pix})$, with most 
of the spectra taken in the wavelength regions $4200-4600 \AA$ or $3800-4900$ $\AA$, respectively.

Some of the stars were also observed at higher resolution with the $1024 \times 1024$ Thomson CCD and cassegrain spectrograph on the 74-inch (1.88-m) telescope of the David Dunlap Observatory. This combination gives approximately $0.2 \AA /$ pix.

\section{Results}

Classification spectra have been taken for approximately 120 of the stars selected by Olsen as having very peculiar uvby indices. Five of these stars are found to have extreme carbon anomalies. One of the most interesting is HD 204848, which is a weak-lined, carbon-rich star with a preliminary classification of K0 III: $\mathrm{Fe}-2.5, \mathrm{CN}-3, \mathrm{CH}+1, \mathrm{Ba}+1$. This classification indicates that it is metal-weak, carbon-strong and nitrogen-weak. There are a few other stars in the sample such as HD 207687 which have similar characteristics, and others with quite different carbon-peculiarities.

HD 204848 and HD 207687 have also been observed with the DDO 1.88m telescope. These DDO spectra from 4000 to $4600 \AA$ are shown in Figure 1, along with similar spectra of the Sun (actually the asteroid Vesta) and the K0 III standard HD 197989.

\section{Discussion}

The advantage of studying G stars is that they are relatively easy to compare to the Sun. In G-type dwarfs the interiors are relatively simple and, in principle, they have not yet suffered any dredge-up to complicate the interpretation of the abundances. However, if they are giants, all is not so simple. Some of the spectra violate our usual concepts of the patterns of spectroscopic peculiarities. HD 204848, for example, exhibits a very strong $\mathrm{CH}$ band with a general weakening of the iron lines. At the same time, it shows virtually no $\mathrm{CN}$, yet other features indicate that it is probably above the main sequence, though it is difficult to be sure (hence the colon) because normally luminosity-sensitive lines of Sr II and Ba II are affected by the same dredge-up process which probably produces the excess carbon, and thus their strength is not a reliable indicator of surface gravity. However, the luminosity class based on the $4376 / 4383$ ratio, which should be relatively independent of abundance, indicates that the star is probably a subgiant or giant.

Photometric temperatures and $\mathrm{CN}$ indices for several of these stars are presented in an accompanying poster (Wing, Garrison \& Koktay 2000). A spectroscopic analysis to determine the model parameters and composition of HD 204848 and HD 207687 is in progress by Koktay. 


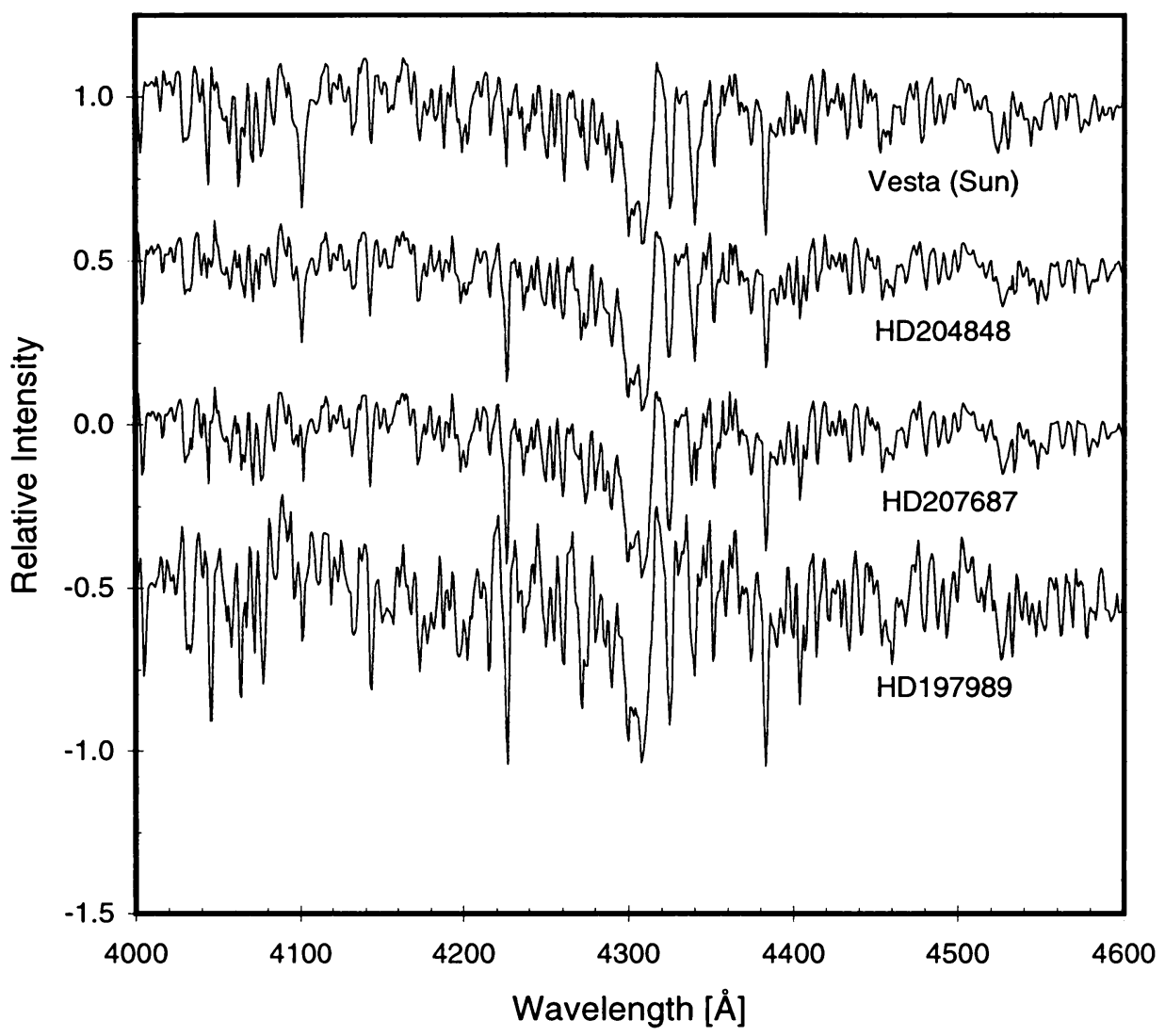

Figure 1. Spectra of normal and carbon-peculiar stars. From top to bottom: (a) Vesta (G2 V), (b) HD 204848 (K0 III: $\mathrm{Fe}-2.5, \mathrm{CN}-3, \mathrm{CH}+1, \mathrm{Ba}+1$ ), (c) HD 207687 (K0 III: $\mathrm{Fe}-2, \mathrm{CN}-3, \mathrm{CH}+2, \mathrm{Ba}+1$ ), and (d) the standard HD 197989 (K0 III). For clarity, the continuum levels of the spectra are offset by 0.5 units, where unity is equal to the difference between zero and pseudo-continuum.

We wish to acknowledge that the research of Koktay has been supported by a TÜBITAK NATO A2 scholarship.

\section{References}

Olsen, E. H. 1993, A\&A Supp., 102, 89

Olsen, E. H. 1994, A\&A Supp., 104, 429

Wing, R.F., Garrison, R.F. \& Koktay, T. 2000, in IAU Symp. 177: The Carbon Star Phenomenon, ed. R. F. Wing (Kluwer), p. 588 